Supplementary Information for

\title{
CRISPR enzyme kinetics for molecular diagnostics
}

\author{
Ashwin Ramachandran and Juan G. Santiago* \\ Department of Mechanical Engineering, Stanford University, California, USA 94305. \\ Email: ashwinre@stanford.edu, juan.santiago@stanford.edu \\ * Corresponding author
}

\section{Contents}

S1. Fluorescence versus cleaved reporter concentration calibration

S2. Michaelis-Menten kinetics measurements for trans-cleavage activity of LbCas 12 a

S3. Model predictions for direct CRISPR-Cas12 detection using gRNA-1

S4. Values of variables used for the back-of-the-envelope checks presented in Tables 1 and 2 S5. List of sequences used in this work

S6. Kinetics parameters for LbCas12a obtained from Michaelis-Menten equation curve fit 


\section{S1. Fluorescence versus cleaved reporter concentration calibration}

Figure S1 shows various steps associated with our signal calibration for CRISPR kinetics measurements. Figure S1a shows background-subtracted fluorescence $F_{c l}$ versus cleaved reporter concentration $c_{c l}$ measured using a thermal cycler (BioRad, MiniOpticon). Background subtraction was carried out by subtracting the signal of a water-only sample from the signal obtained from titrated quantities of fully cleaved ssDNA reporters. Reporters were pre-cleaved by subjecting them to the trans-cleavage reaction of activated LbCas12a-gRNA-1. For this, $100 \mathrm{nM}$ of activated enzyme (activated using dsDNA target) was mixed with varying reporter concentrations between $31.25 \mathrm{nM}$ and $2000 \mathrm{nM}$ with a final volume of $30 \mu \mathrm{L}$. The trans-cleavage reaction was performed at $37^{\circ} \mathrm{C}$ for $\sim 10 \mathrm{~h}$. At the end of this pre-cleavage step, we verified that the fluorescence signal of each reaction was constant in time. Shown in Figure S1a is the measured, background-subtracted, steady-state (fully cleaved) fluorescence signal $F_{c l}$ versus cleaved reporter concentration $c_{c l}$, obtained using the aforementioned procedure. We obtained a linear fit to the data in Figure S1a given by $F_{c l}=0.00334 c_{c l}$ where $c_{c l}$ is the cleaved reporter concentration in $\mathrm{nM}$ and $F_{c l}$ is the arbitrary fluorescence units of our thermal cycler.

Figure S1b shows background-subtracted fluorescence $F_{u c l}$ versus uncleaved reporter concentration $c_{u c l}$. As in Figure S1a, background subtraction here refers to subtracting the signal obtained from a water-only sample from the signal obtained from titrated quantities of uncleaved reporters. A linear fit was obtained to the data in Figure S1b as $F_{u c l}=0.00014 c_{u c l}$, where $c_{u c l}$ is the uncleaved reporter concentration in $\mathrm{nM}$ and $F_{u c l}$ is the arbitrary fluorescence units of our thermal cycler.

In the next section, we describe the procedure used to estimate reaction velocities based on the calibration curves presented in Figure S1a and S1b, and the procedure to convert raw fluorescence data to cleaved reporter concentration.
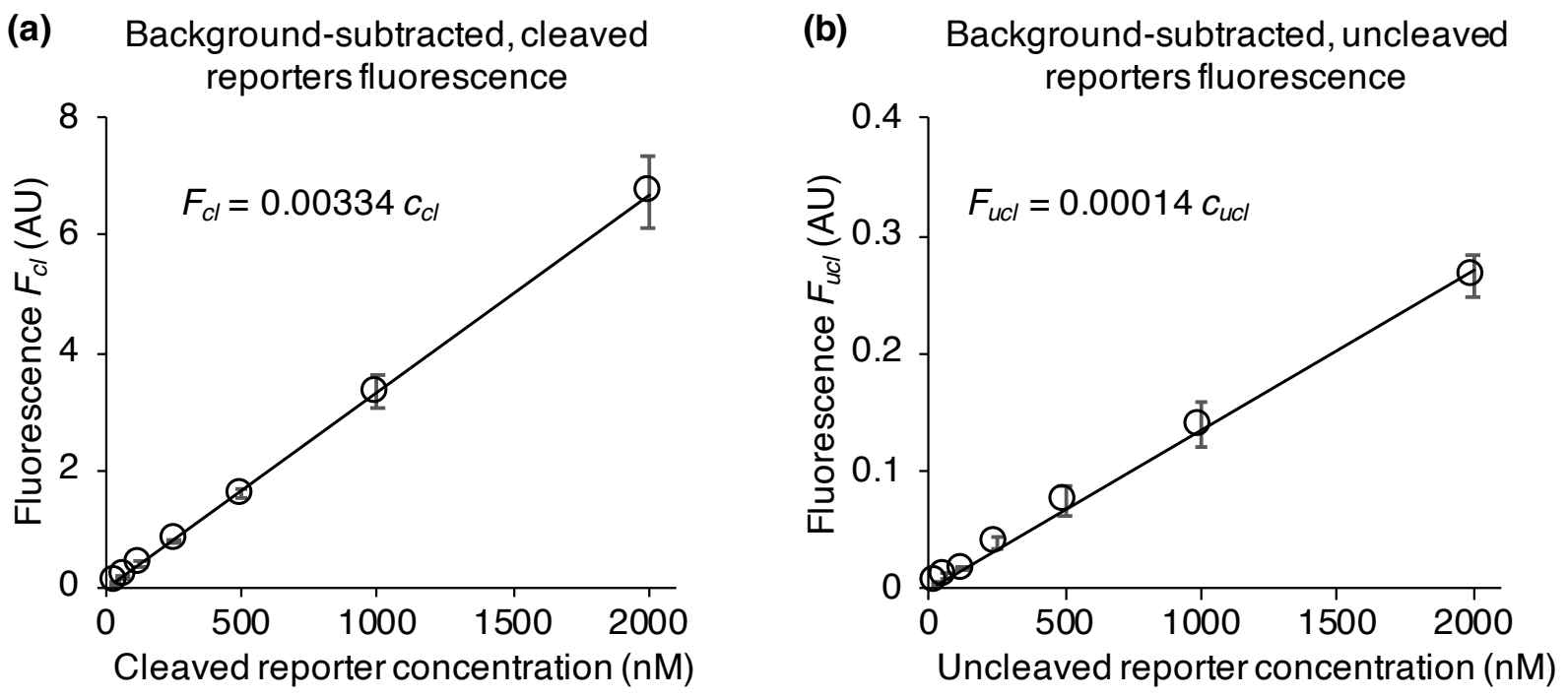

Figure S1. (a) Background-subtracted fluorescence $F_{c l}$ versus concentration of cleaved reporters $c_{c l}$, (b) background-subtracted fluorescence $F_{u c l}$ versus concentration of uncleaved reporters $c_{u c l}$, for the thermal cycler detector. Solid lines are linear regression fits to experimental data (in 
symbols). Three replicates were measured for each concentration. Uncertainty bars indicate one standard deviation.

\section{S2. Michaelis-Menten kinetics measurements for trans-cleavage activity of LbCas12a}

Background-subtracted fluorescence $F$ versus time for trans-cleavage were performed as Michaelis-Menten kinetics experiments. We obtained these using $1 \mathrm{nM}$ of trans-active LbCas $12 \mathrm{a}$ and varying reporter concentrations between $31.25 \mathrm{nM}$ and $2000 \mathrm{nM}$ in a $30 \mu \mathrm{L}$ reaction volume. trans-cleavage kinetics for LbCas12a were studied for two different gRNA sequences (gRNA-1 and gRNA-2) using the corresponding ssDNA and dsDNA activators (Table S3).

Below we describe the procedure used to obtain reaction velocities in $\mathrm{nM} / \mathrm{s}$ from fluorescence versus time data of Michaelis-Menten kinetics experiments. We hypothesize that the background-subtracted fluorescence as a function of time $F(t)$ measured during trans-cleavage experiment is the sum of fluorescence from cleaved reporters $F_{c l}(t)$ and uncleaved reporters $F_{u c l}(t)$. Note that the quenching of a fluorophore in an intact reporter is imperfect. For example, from Figures S1a and S1b, at concentration of $2000 \mathrm{nM}$, we observed a ratio cleaved-to-uncleaved fluorescence ratio of $\sim 24$ for our reporters. Thus, we write

$$
F(t)=F_{c l}(t)+F_{u c l}(t) .
$$

Substituting the calibration curve equations from Figure S1 in Equation (1), we obtain

$$
F(t)=0.00334 c_{c l}(t)+0.00014 c_{u c l}(t) .
$$

From mass conservation, $c_{c l}(t)+c_{u c l}(t)=c_{0}$, where $c_{0}$ is the initial concentration of uncleaved reporters. Thus, Equation (2) can be rewritten as

$$
F(t)=0.00334 c_{c l}(t)+0.00014\left(c_{0}-c_{c l}(t)\right)=0.0032 c_{c l}(t)+0.00014 c_{0} .
$$

The reaction velocities $d c_{c l} / d t$ in $\mathrm{nM} / \mathrm{s}$ is obtained by differentiating Equation (3) with respect to time as

$$
\frac{d c_{c l}}{d t}=\frac{1}{0.0032} \times \frac{d F}{d t}
$$

Lastly, the cleaved reporter concentration $c_{c l}$ (in $\mathrm{nM}$ ) versus time is estimated using Equation (3) as

$$
c_{c l}(t)\left(\text { in nM) }=\frac{F(t)-0.00014 c_{0}}{0.0032}=\frac{F(t)-F_{u c l}\left(c_{0}\right)}{0.0032} .\right.
$$

Figures S2 and S3 show background-subtracted fluorescence $F$ versus time for transactive Cas12-gRNA-1 and Cas12-gRNA-2, respectively, which were activated using ssDNA and dsDNA targets. Reaction velocities (in $\mathrm{nM} / \mathrm{s}$ ) versus substrate (reporter) concentration obtained from the linear fits in Figures $\mathbf{S 2}$ and $\mathbf{S 3}$ are shown in Figure $\mathbf{2}$ of the main manuscript. 
(a)

gRNA-1 (dsDNA activator)

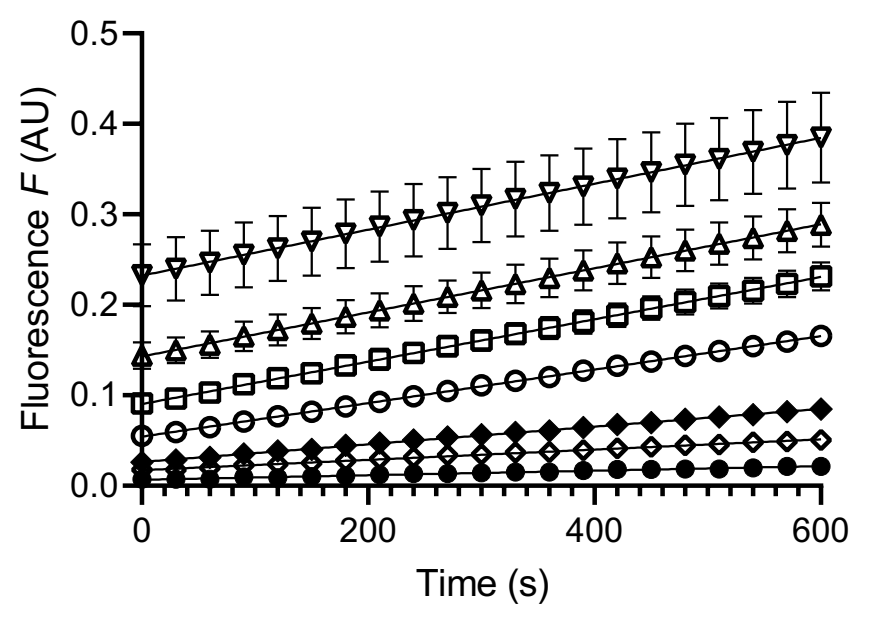

$$
\begin{aligned}
& \bullet 31.25 \mathrm{nM} \\
& \bullet 62.5 \mathrm{nM} \\
& \bullet 125 \mathrm{nM} \\
& -250 \mathrm{nM} \\
& \bullet 500 \mathrm{nM} \\
& -1000 \mathrm{nM} \\
& \rightarrow 2000 \mathrm{nM}
\end{aligned}
$$

(b)

gRNA-1 (ssDNA activator)

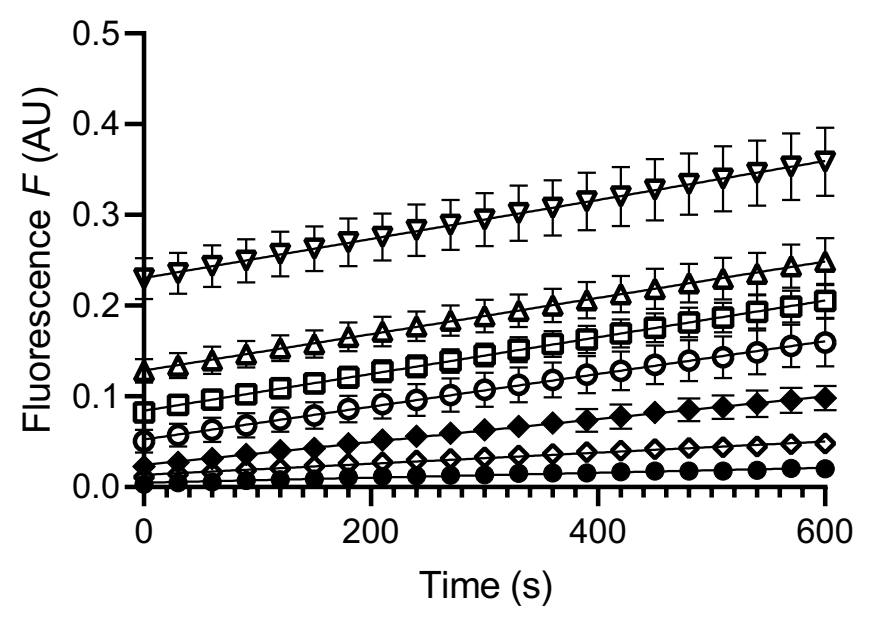

$-31.25 \mathrm{nM}$

$\rightarrow 62.5 \mathrm{nM}$

$\rightarrow 125 \mathrm{nM}$

- $250 \mathrm{nM}$

๑ $500 \mathrm{nM}$

$\triangle 1000 \mathrm{nM}$

$\rightarrow 2000 \mathrm{nM}$

Figure S2. Raw measurements of fluorescence signal associated with trans-cleavage kinetics experiments for target 1. Both ssDNA and dsDNA forms of this target sequence were used using LbCas 12a. Background-subtracted fluorescence versus time for varying concentration of reporters between $31.25 \mathrm{nM}$ and $2000 \mathrm{nM}$, and for $1 \mathrm{nM}$ of Cas 12 enzyme activated by (a) dsDNA and (b) ssDNA activators. Three replicates were measured for each concentration. Solid lines are linear regression fits to the background-subtracted experimental data (symbols). Uncertainty bars indicate one standard deviation. Slopes of linear regression fits were used to estimate reaction velocities (Figure 2a of the main paper). 
(a)

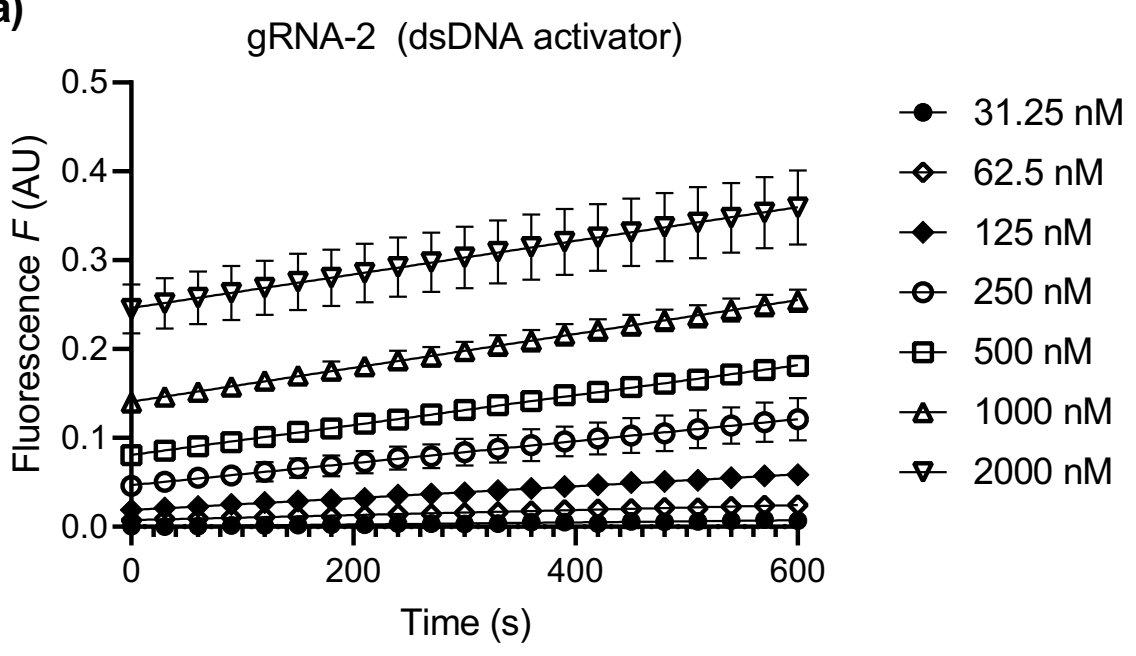

(b)

gRNA-2 (ssDNA activator)

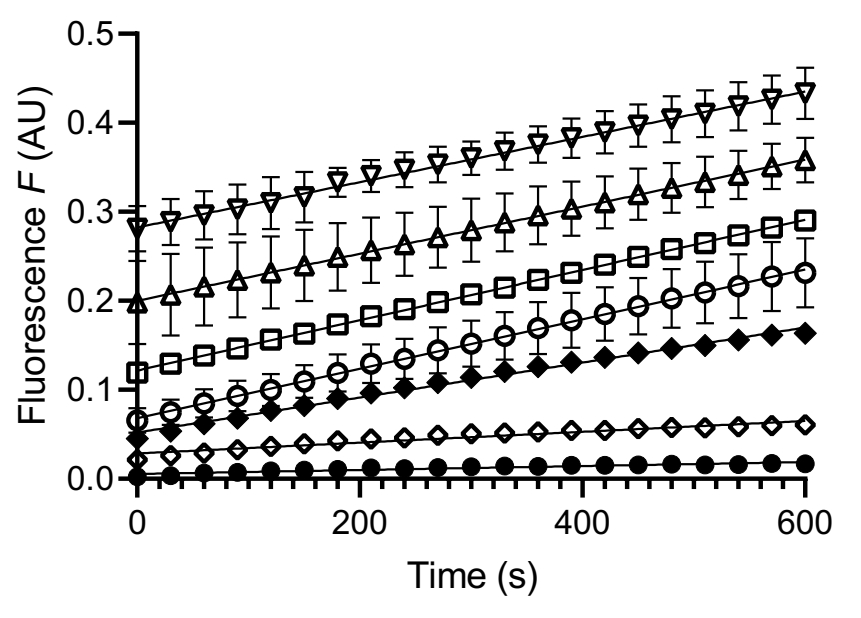

-- $31.25 \mathrm{nM}$

$\rightarrow 62.5 \mathrm{nM}$

$\bullet 125 \mathrm{nM}$

- $250 \mathrm{nM}$

४ $500 \mathrm{nM}$

$\triangle 1000 \mathrm{nM}$

$\rightarrow 2000 \mathrm{nM}$

Figure S3. Raw measurements of fluorescence signal associated with trans-cleavage kinetics experiments for target 2. Both ssDNA and dsDNA forms of this target sequence were used using LbCas12a. Background-subtracted fluorescence versus time for varying concentration of reporters between $31.25 \mathrm{nM}$ and $2000 \mathrm{nM}$, and for $1 \mathrm{nM}$ of Cas 12 enzyme activated by (a) dsDNA and (b) ssDNA activators. Three replicates were measured for each concentration. Solid lines are linear regression fits to experimental data (in symbols). Uncertainty bars indicate one standard deviation. Slopes of linear regression fits were used to estimate reaction velocities (Figure $\mathbf{2 b}$ of the main paper). 


\section{S3. Model predictions for direct CRISPR-Cas12 detection using gRNA-1}

Figure S4 shows contours of the predicted cleaved reporter fraction (ratio of cleaved and total reporter concentration; a measure of the signal to noise ratio) for varying target concentration between $10 \mathrm{fM}$ and $10 \mathrm{nM}$, and initial reporter concentration between $1 \mathrm{nM}$ and $10 \mathrm{uM}$. For these predictions, we considered the estimates of $k_{c a t}$ and $K_{M}$ obtained from our own experiments, namely $0.09 \mathrm{~s}^{-1}$ and $213 \mathrm{nM}$, respectively, corresponding to LbCas12a-gRNA-1 with dsDNA activator (Table 1). Shown are cleaved reporter fraction values after $60 \mathrm{~min}$ of assay time. Note here that the $k_{\text {cat }} / K_{M}$ is $4.2 \times 10^{5} \mathrm{M}^{-1} \mathrm{~s}^{-1}$. For comparison, Figure 5 of the main manuscript showed a similar plot, but assumed a $k_{\text {cat }} / K_{M}$ of $1.7 \times 10^{7} \mathrm{M}^{-1} \mathrm{~s}^{-1}$ and this higher number corresponds to the updated value for LbCas 12a with dsDNA activator from Chen et al. (2018). ${ }^{1,2}$ From Figures 5 and S4, we observe that a lower $k_{\text {cat }} / K_{M}$ significantly lowers the degree of reaction completion. For example, from Figure S4 $\left(k_{c a t} / K_{M}\right.$ is $\left.4.2 \times 10^{5} \mathrm{M}^{-1} \mathrm{~s}^{-1}\right)$, less than $1 \%$ of the initial reporters are cleaved for target concentrations of $10 \mathrm{pM}$ and lower. In contrast, from Figure 5, $\left(k_{\text {cat }} / K_{M}\right.$ is $1.7 \times 10^{7} \mathrm{M}^{-1} \mathrm{~s}^{-1}$ ), less than $1 \%$ of the initial reporters are cleaved for target concentrations of $\sim 200$ $\mathrm{fM}$ and lower. Note we assume here that $1 \%$ of cleaved reporters as an optimistic threshold which would be required for highly sensitive detectors to produce a measurable signal above a noise floor. The realistic limits of detection for LbCas12a (for order $1 \mathrm{~h}$ assay times) given these estimates of kinetic rates are likely to be in the range of $100 \mathrm{fM}$ to $1 \mathrm{pM}$ depending on the catalytic efficiency of the enzyme-target pair. We further hypothesize similar limits of detection for CRISPR-Cas 13 based on the updated kinetic parameters from presented in Table 2.

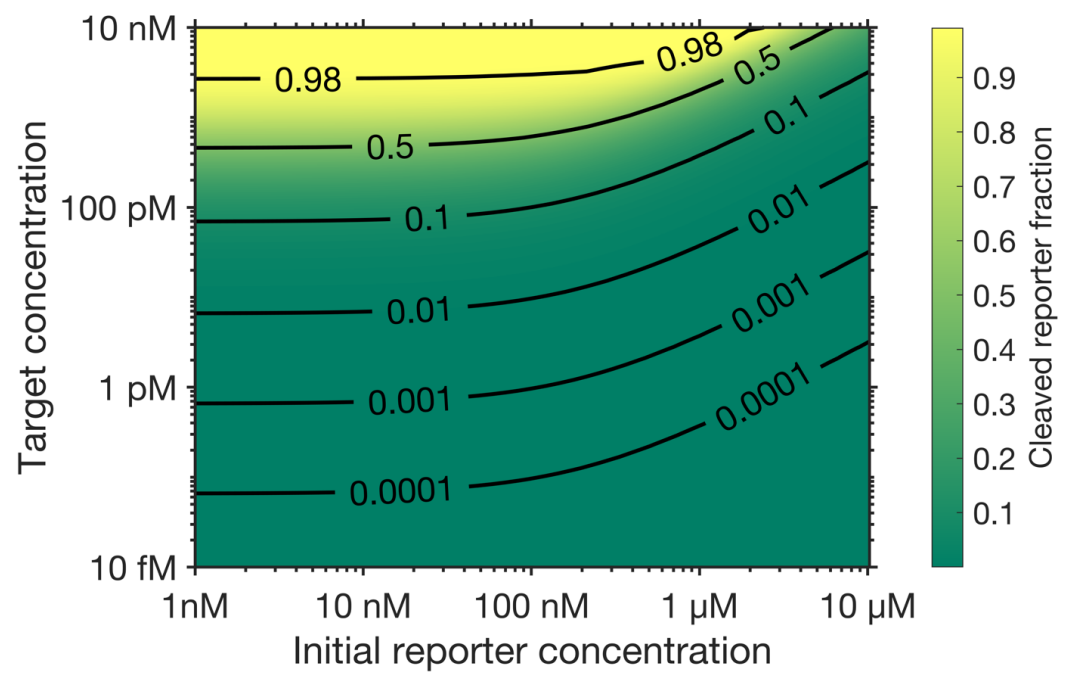

Figure S4. Model predictions for the fraction of cleaved reporters $[P]$ relative to the initial amount of uncleaved reporters $S_{0}$. Contours of the cleaved reporter fraction are plotted as a function of target nucleic acid concentration and initial uncleaved reporter concentration, for a 60 min assay time, and $k_{\text {cat }}$ and $K_{M}$ of $0.09 \mathrm{~s}^{-1}$ and $2.13 \times 10^{-7} \mathrm{M}$, respectively (corresponding to LbCas 12-gRNA1 activated by dsDNA). Contour levels of 0 and 1 represent no reporter cleavage and complete reporter cleavage, respectively. 


\section{S4. Values of variables used for the back-of-the-envelope checks presented in Tables 1 and 2}

Table S1. List of values of variables and figure/table references used in our back-of-the-envelope checks for CRISPR-Cas12 and CRISPR-Cas13 based on data presented in the original publications. ${ }^{1,3-7}$ See Table 1 for the calculated $\alpha, \beta$, and $\gamma$ values.

\begin{tabular}{|c|c|c|c|c|c|c|c|c|}
\hline \multirow{2}{*}{$\begin{array}{l}\text { CRISPR enzyme and } \\
\text { activator }\end{array}$} & \multicolumn{3}{|c|}{$\begin{array}{c}\text { Originally reported kinetic } \\
\text { parameters }\end{array}$} & \multicolumn{4}{|c|}{ Values used for the back-of-the-envelope checks } & \multirow{2}{*}{ Original references } \\
\hline & $k_{c a t}(1 / s)$ & $K_{M}(\mathbf{M})$ & $\begin{array}{c}k_{c a t} / K_{M} \\
(1 / \mathbf{M s})\end{array}$ & $v(\mathrm{nM} / \mathrm{s})$ & $t_{\text {lin }}(\mathbf{s})$ & $S_{0}(\mathrm{nM})$ & $E_{0}(\mathbf{n M})$ & \\
\hline LbCas $12 \mathrm{a}$ (ssDNA activator) & 250 & $4.94 \times 10^{-7}$ & $5.1 \times 10^{8}$ & 17 & 1,380 & 2,000 & 0.1 & $\begin{array}{l}\text { Figures 2D and S7 - Chen et al. } \\
\qquad(2018)\end{array}$ \\
\hline LbCas12a (dsDNA activator) & 1250 & $7.25 \times 10^{-7}$ & $1.7 \times 10^{9}$ & 75 & 390 & 2,000 & 0.1 & $\begin{array}{l}\text { Figures 2D and S7 - Chen et al. } \\
\qquad(2018)\end{array}$ \\
\hline AsCas12a (dsDNA activator) & 0.6 & $2.7 \times 10^{-6}$ & $2.4 \times 10^{5}$ & 5.2 & 180 & 15,000 & 10 & $\begin{array}{c}\text { Appendix 2, figure 1, figure } \\
\text { supplement } 2 \text { - Cofsky et al. (2020) }\end{array}$ \\
\hline $\begin{array}{c}\text { PbuCas13b (ssRNA } \\
\text { activator) }\end{array}$ & 987 & - & - & $\sim 120,000$ & 1,200 & 6,400 & 5 & Figure 5D, Slaymaker et al. (2019) \\
\hline $\begin{array}{c}\text { LbuCas13a (ssRNA } \\
\text { activator) }\end{array}$ & 4854 & $4.45 \times 10^{-6}$ & $1.1 \times 10^{9}$ & 360 & 600 & 10,000 & 0.01 & $\begin{array}{l}\text { Figure 3, Results and Discussion } \\
\text { Section - Shan et al. (2019) }\end{array}$ \\
\hline $\begin{array}{l}\text { LbuCas13a (ssRNA } \\
\text { activator) }\end{array}$ & 204 & $2.62 \times 10^{-6}$ & $0.8 \times 10^{8}$ & 150 & 600 & 10,000 & 1 & Figure S3, Zhou et al. (2020) \\
\hline $\begin{array}{l}\text { LbCas12a (wild-type crRNA, } \\
\text { dsDNA activator) }\end{array}$ & 1098 & $6.86 \times 10^{-7}$ & $1.6 \times 10^{9}$ & 600 & 1,800 & 2,000 & 7.4 & $\begin{array}{c}\text { Figure 2E and Supplementary Figure } \\
\text { 5, Nguyen et al. (2020) }\end{array}$ \\
\hline $\begin{array}{c}\text { LbCas12a (crRNA + } \\
\text { 3'DNA7, dsDNA activator) }\end{array}$ & 2189 & $4.29 \times 10^{-7}$ & $5.1 \times 10^{9}$ & 1,450 & 1,800 & 2,000 & 7.4 & $\begin{array}{c}\text { Figure 2E and Supplementary Figure } \\
\text { 5, Nguyen et al. (2020) }\end{array}$ \\
\hline $\begin{array}{l}\text { LbCas12a (dsDNA activator: } \\
\text { gRNA-1, gRNA-2) }\end{array}$ & $\begin{array}{l}0.09 \\
0.07\end{array}$ & $\begin{array}{l}2.13 \times 10^{-7}, \\
2.74 \times 10^{-7}\end{array}$ & $\begin{array}{l}4.2 \times 10^{5} \\
2.5 \times 10^{5}\end{array}$ & $\begin{array}{l}0.08 \\
0.06\end{array}$ & 600 & 2,000 & 1 & This work \\
\hline $\begin{array}{l}\text { LbCas12a (ssDNA activator: } \\
\text { gRNA-1, gRNA-2) }\end{array}$ & $\begin{array}{l}0.07 \\
0.09\end{array}$ & $\begin{array}{l}1.27 \times 10^{-7} \\
0.96 \times 10^{-7}\end{array}$ & $\begin{array}{l}5.8 \times 10^{5} \\
8.9 \times 10^{5}\end{array}$ & $\begin{array}{l}0.07 \\
0.08\end{array}$ & 600 & 2,000 & 1 & This work \\
\hline
\end{tabular}


Table S2. List of updated variables used in our back-of-the-envelope checks for CRISPR-Cas 12 and CRISPR-Cas 13 based on data presented in the two recently published errata. ${ }^{2,8}$ See Table 2 for the corresponding $\alpha, \beta$, and $\gamma$ values.

\begin{tabular}{|c|c|c|c|c|c|c|c|c|}
\hline \multirow{2}{*}{$\begin{array}{l}\text { CRISPR enzyme } \\
\text { and activator }\end{array}$} & \multicolumn{3}{|c|}{ Updated kinetic parameters } & \multicolumn{4}{|c|}{ Values used for the back-of-the-envelope checks } & \multirow{2}{*}{ References } \\
\hline & $k_{c a t}(1 / s)$ & $K_{M}(\mathbf{M})$ & $\begin{array}{c}\boldsymbol{k}_{c a t} / K_{M} \\
(\mathbf{1} / \mathbf{M s})\end{array}$ & $v(\mathrm{nM} / \mathrm{s})$ & $t_{\text {lin }}(\mathbf{s})$ & $S_{0}(\mathrm{nM})$ & $E_{0}(\mathrm{nM})$ & \\
\hline $\begin{array}{c}\mathrm{LbCas} 12 \mathrm{a}(\mathrm{ssDNA} \\
\text { activator) }\end{array}$ & 2 & $6.2 \times 10^{-7}$ & $5 \times 10^{6}$ & 0.22 & 390 & 2,000 & 0.1 & $\begin{array}{l}\text { Figures 2D and S7 - Correction: } \\
\text { Chen et al. (2021) }\end{array}$ \\
\hline $\begin{array}{l}\mathrm{LbCas} 12 \mathrm{a}(\mathrm{dsDNA} \\
\text { activator) }\end{array}$ & 17 & $1.01 \times 10^{-6}$ & $1.7 \times 10^{7}$ & 1.1 & 390 & 2,000 & 0.1 & $\begin{array}{l}\text { Figures 2D and S7 - Correction: } \\
\text { Chen et al. (2021) }\end{array}$ \\
\hline $\begin{array}{c}\text { PbuCas } 13 \mathrm{~b} \\
\text { (ssRNA activator) }\end{array}$ & 0.95 & $10.86 \times 10^{-6}$ & $9.2 \times 10^{4}$ & 1.8 & 1,200 & 6,400 & 5 & Correction: Slaymaker et al. (2021) \\
\hline
\end{tabular}




\section{S5. List of sequences used in this work}

Table S3. List of gRNAs, template (TS) and non-template (NTS) ssDNA, and reporter ssDNA sequences used for trans-cleavage kinetics measurements of LbCas12a. dsDNA activators were prepared by annealing TS and NTS (see Experimental Section of the main paper).

\begin{tabular}{|c|c|c|}
\hline Sequence name & $\begin{array}{c}\text { RNA/DNA } \\
\text { (vendor) }\end{array}$ & Sequence (5'-3') \\
\hline gRNA-1 (target 1) & RNA (PAN) & $\begin{array}{l}\text { UAAUUUCUACUAAGUGUAGAUUGUAUGGCA } \\
\text { UGAGUAACGAA }\end{array}$ \\
\hline NTS (target 1) & DNA (Elim) & $\begin{array}{l}\text { TCTATCCATTTATGTATGGCATGAGTAACGAA } \\
\text { GAATATAAT }\end{array}$ \\
\hline TS (target 1 ) & DNA (Elim) & $\begin{array}{l}\text { ATTATATTCTTCGTTACTCATGCCATACATAAA } \\
\text { TGGATAGA }\end{array}$ \\
\hline gRNA-2 (target 2) & RNA (PAN) & $\begin{array}{l}\text { UAAUUUCUACUAAGUGUAGAUGUGGUAUUC } \\
\text { UUGCUAGUUAC }\end{array}$ \\
\hline NTS (target 2) & DNA (Elim) & $\begin{array}{l}\text { TTTTTCTTGCTTTCGTGGTATTCTTGCTAGTTAC } \\
\text { ACTAGCCATCCTTACTGCGCTTCGAT }\end{array}$ \\
\hline TS (target 2) & DNA (Elim) & $\begin{array}{l}\text { ATCGAAGCGCAGTAAGGATGGCTAGTGTAAC } \\
\text { TAGCAAGAATACCACGAAAGCAAGAAAAA }\end{array}$ \\
\hline $8 \mathrm{nt}$ ssDNA reporter & DNA (PAN) & /56-FAM/TTATTATT/BHQ-1 \\
\hline
\end{tabular}

\section{S6. Kinetics parameters for LbCas12a obtained from Michaelis-Menten equation curve fit}

Table S4. Kinetic parameters $k_{c a t}$ and $K_{M}$ obtained from Michaelis-Menten equation curve fits to experimental data shown in Figure 2. Listed below are best fit values and the $95 \%$ confidence interval range of these parameters obtained from GraphPad Prism software (CA, USA).

\begin{tabular}{|c|c|c|c|c|}
\hline \multirow{2}{*}{ gRNA/activator } & \multicolumn{2}{|c|}{ Best fit } & \multicolumn{2}{|c|}{$95 \%$ confidence interval } \\
\hline & $k_{\text {cat }}\left(\mathrm{s}^{-1}\right)$ & $K_{M}(\mathrm{nM})$ & $k_{c a t}\left(\mathrm{~s}^{-1}\right)$ & $K_{M}(\mathrm{nM})$ \\
\hline gRNA-1 (dsDNA) & 0.089 & 213 & 0.081 to 0.098 & 159 to 284 \\
\hline gRNA-1 (ssDNA) & 0.073 & 128 & 0.068 to 0.079 & 98 to 165 \\
\hline gRNA-2 (dsDNA) & 0.069 & 274 & 0.062 to 0.077 & 198 to 381 \\
\hline gRNA-2 (ssDNA) & 0.086 & 96 & 0.079 to 0.093 & 69 to 131 \\
\hline
\end{tabular}




\section{References}

(1) Chen, J. S.; Ma, E.; Harrington, L. B.; Da Costa, M.; Tian, X.; Palefsky, J. M.; Doudna, J. A. CRISPR-Cas12a Target Binding Unleashes Indiscriminate Single-Stranded DNase Activity. Science (80-. ). 2018, 360 (6387), 436-439.

(2) Erratum for the Report "CRISPR-Cas12a Target Binding Unleashes Indiscriminate Single-Stranded DNase Activity" by J. S. Chen, E. Ma, L. B. Harrington, M. Da Costa, X. Tian, J. M. Palefsky, J. A. Doudna. Science (80-. ). 2021, 371 (6531), eabh0317.

(3) Cofsky, J. C.; Karandur, D.; Huang, C. J.; Witte, I. P.; Kuriyan, J.; Doudna, J. A. CRISPR-Cas12a Exploits R-Loop Asymmetry to Form Double-Strand Breaks. Elife 2020, 9 .

(4) Slaymaker, I. M.; Mesa, P.; Kellner, M. J.; Kannan, S.; Brignole, E.; Koob, J.; Feliciano, P. R.; Stella, S.; Abudayyeh, O. O.; Gootenberg, J. S.; et al. High-Resolution Structure of Cas13b and Biochemical Characterization of RNA Targeting and Cleavage. Cell Rep. 2019, 26 (13), 3741-3751.e5.

(5) Zhou, T.; Huang, R.; Huang, M.; Shen, J.; Shan, Y.; Xing, D. CRISPR/Cas13a Powered Portable Electrochemiluminescence Chip for Ultrasensitive and Specific MiRNA Detection. Adv. Sci. 2020, 7 (13), 1903661.

(6) Shan, Y.; Zhou, X.; Huang, R.; Xing, D. High-Fidelity and Rapid Quantification of MiRNA Combining CrRNA Programmability and CRISPR/Cas13a Trans -Cleavage Activity. Anal. Chem. 2019, 91 (8), 5278-5285.

(7) Nguyen, L. T.; Smith, B. M.; Jain, P. K. Enhancement of Trans-Cleavage Activity of Cas12a with Engineered CrRNA Enables Amplified Nucleic Acid Detection. Nat. Commun. 2020, 11 (1), 4906.

(8) Slaymaker, I. M.; Mesa, P.; Kellner, M. J.; Kannan, S.; Brignole, E.; Koob, J.; Feliciano, P. R.; Stella, S.; Abudayyeh, O. O.; Gootenberg, J. S.; et al. High-Resolution Structure of Cas $13 \mathrm{~b}$ and Biochemical Characterization of RNA Targeting and Cleavage. Cell Rep. 2021, 34 (10), 108865. See Erratum of March 9, 2021 